\title{
Evidence-based treatment of open ankle fractures
}

\author{
Caroline C. C. Hulsker · Sanne Kleinveld • \\ Chris B. L. Zonnenberg • Mike Hogervorst • \\ Michel P. J. van den Bekerom
}

Received: 5 April 2011 / Published online: 29 June 2011

(C) The Author(s) 2011. This article is published with open access at Springerlink.com

\begin{abstract}
Fractures of the ankle are fairly common injuries. Open ankle fractures are much less common and associated with severe injuries to surrounding tissues. We have performed a systematic review of the literature concerning the clinical results and complication rates in the treatment of open ankle fractures. We conducted a search limited to the following databases: Pubmed/Medline, Cochrane Database of Systematic Reviews, Cochrane Clinical Trial Register and Embase. These were searched from 1968 to April 2010 to identify studies relating to the treatment of open ankle fractures. Fifteen articles concerning 498 patients with treatment of an open ankle fracture were identified. The number of included patients varied from 11 to 64 . There were 2 prospective and 13 retrospective studies. All articles were case series and classified as Level IV evidence. In 373 cases, open ankle fractures were treated by immediate internal fixation. In 125 cases, a conservative treatment or delayed/other fixation treatment was followed. Of those patients treated by immediate internal fixation, $81 \%$ had satisfactory result. Poor results $(15 \%)$ were most
\end{abstract}

C. C. C. Hulsker $(\square) \cdot$ S. Kleinveld

Department of General Surgery, Academic Medical Centre, P.O. Box 22660, Amsterdam DD 1100, The Netherlands

e-mail: c.hulsker@gmail.com

C. B. L. Zonnenberg

Department of Orthopaedics and Trauma, Spaarne Hospital, Hoofddorp, The Netherlands

M. Hogervorst

Department of General Surgery, Gelre Hospitals,

Apeldoorn, The Netherlands

M. P. J. van den Bekerom

Department of Orthopaedic Surgery, Academic Medical Centre,

P.O. Box 22660, Amsterdam DD 1100, The Netherlands commonly due to non-anatomic reductions, articular surface damage or deep infection. When conservative treatment was followed, $76 \%$ had satisfactory results. The most reported complications after immediate internal fixation were deep infection (8\%) and skin necrosis (14\%). There is a lack of high quality literature concerning the (operative) treatment of patients with open ankle fractures. Remarkable is that most authors reported satisfactory results after performance of their treatment protocol. Based on the available literature, we formulated guidelines regarding: timing of operative treatment, wound irrigation, the role of internal fixation, wound coverage and closure, the use of antibiotics and additional therapies.

Keywords Ankle fractures - Open/complex/compound · Treatment $\cdot$ Osteosynthesis $\cdot$ Operative procedures .

Antibiotics

\author{
Abbreviations \\ AO/ASIFArbeitsgemeinschaft für Osteosynthesefragen/ \\ American Society for Internal Fixation \\ OTA Orthopaedic Trauma Association
}

\section{Introduction}

In the treatment of open fractures, the surgeon's objectives are to prevent infection, promote fracture healing, and restore function without complications. [1]. A fracture is considered to be open when disruption of the skin and underlying soft tissues results in a communication between the fracture and the outside environment. The most commonly used classification for open fractures is the system developed by Gustilo and Anderson [2], and subsequently modified by Gustilo et al. [3] (Table 1). 
Table 1 Classification system of Gustilo and Anderson [3, 4]

I Wound $<1 \mathrm{~cm}$; minimal contamination, comminution and soft tissue damage

II Wound $>1 \mathrm{~cm}$; moderate soft tissue damage, minimal periosteal stripping

IIIA Severe soft tissue damage and substantial contamination; coverage adequate

IIIB Severe soft tissue damage and substantial contamination; coverage inadequate

IIIC Arterial injury requiring repair

Two studies found the Gustilo and Anderson classification system to be associated with low interobserver agreement $[4,5]$ In spite of these limitations, the Gustilo and Anderson classification remains the preferred system for categorizing open fractures since the fracture type correlates well with the risk of infection and other associated complications.

Fractures of the ankle are fairly common injuries. Open ankle fractures are much less common and usually caused by high-energy trauma. In most series, an incidence of about 5\% is reported [6]. The amount of energy in the initial trauma determines the type of injury and prognosis. Significant bone and soft tissue loss, either from the initial injury or subsequent debridement, can create difficulties in obtaining wound closure, joint congruity and fracture union. It is not surprising, therefore, that these fractures often end in poor results.

Okike and Bhattacharyya already formulated guidelines for treatment of open fractures in general [1]. The objective of this article is to formulate guidelines for treatment of open ankle fractures. These guidelines are based on a systematic review of the literature. The aim was to evaluate the clinical results and complication rates after different (non) operative treatment protocols. Based on these results, recommendations for clinical practice and future research were formulated. Principles for treatment of closed ankle fractures are not discussed in this review.

\section{Materials and methods}

For inclusion and exclusion criteria, we refer to Fig. 1. The following search terms were used: open/compound/complex fractures, ankle/malleolar fractures, treatment outcome, osteosynthesis, debridement, lavage, irrigation, antibiotic treatment and operative treatment. With the help of a clinical librarian, a search limited to adult humans was conducted in the following databases: Pubmed/Medline, Cochrane Database of Systematic Reviews, Cochrane Clinical Trial Register, Current Controlled Trials, Orthopedic Trauma Association (OTA) annual meetings' abstracts archives website and Embase. These databases were searched from 1968 to April 2010 to identify studies relating to the (non) operative treatment of open ankle fractures. Review articles and expert opinions were excluded because these articles do not report on new patient series. Also, reports on surgical techniques and abstracts from scientific meetings were excluded. Furthermore, the lists of references of retrieved publications were manually checked for additional studies potentially meeting the inclusion criteria and not found by the electronic search. The search was restricted to articles written in the English, French, German and Dutch language.

The search of the literature in this study was performed according to the Quorom [7] statement on meta-analyses and limited to published original studies including adult patients with (non) operative treatment of open ankle fractures (AO/ASIF type 44ABC).

All operative techniques and non-operative treatment modalities were included. The diagnosis of an open ankle fracture was made based on history, physical examination and standard radiography. Only studies with a minimum of seven patients were included. Articles concerning the treatment of a distal tibial fracture or a tibial pilon fracture $(\mathrm{AO} /$ ASIF type 43 A, B, C) were not included. Articles concerning open and closed fractures were included when the open ankle fractures could be analyzed separately. The Pubmed search strategy is shown in Table 2.

Relevant baseline measurements were: use of antibiotics, timing of treatment/debridement, use of irrigation, type of fixation, coverage and closure, and use of adjunctive therapies. After an (non) operatively treated open ankle fracture, relevant outcome measures of the included studies were: functional outcome (different scoring systems were used in the reviewed articles), radiographic osteoarthritis, rate of non/malunion, rate of infection, performing wound culture, rate of secondary surgical interventions. The open ankle fractures were classified according to the system developed by Gustilo and Anderson [2], and subsequently modified by Gustilo et al. [3].

A wound or deep infection is defined as an invasion of the joint or the soft tissues around the joint by pathogenic microorganisms. The definition of malunion was documented for each article. The rate of malunion according to the authors' definition was extracted from each article. The non-union rate included those fractures that developed osseous non-union after 1-year follow-up. Delayed unions were not included in the non-union rate if the fractures subsequently healed. The secondary surgical procedure rate included any reported secondary surgical procedure related to the ankle fracture. This also included partial and complete hardware removals.

Two reviewers $(\mathrm{CH}, \mathrm{MB})$ independently reviewed the literature searches on title and abstract to identify relevant 


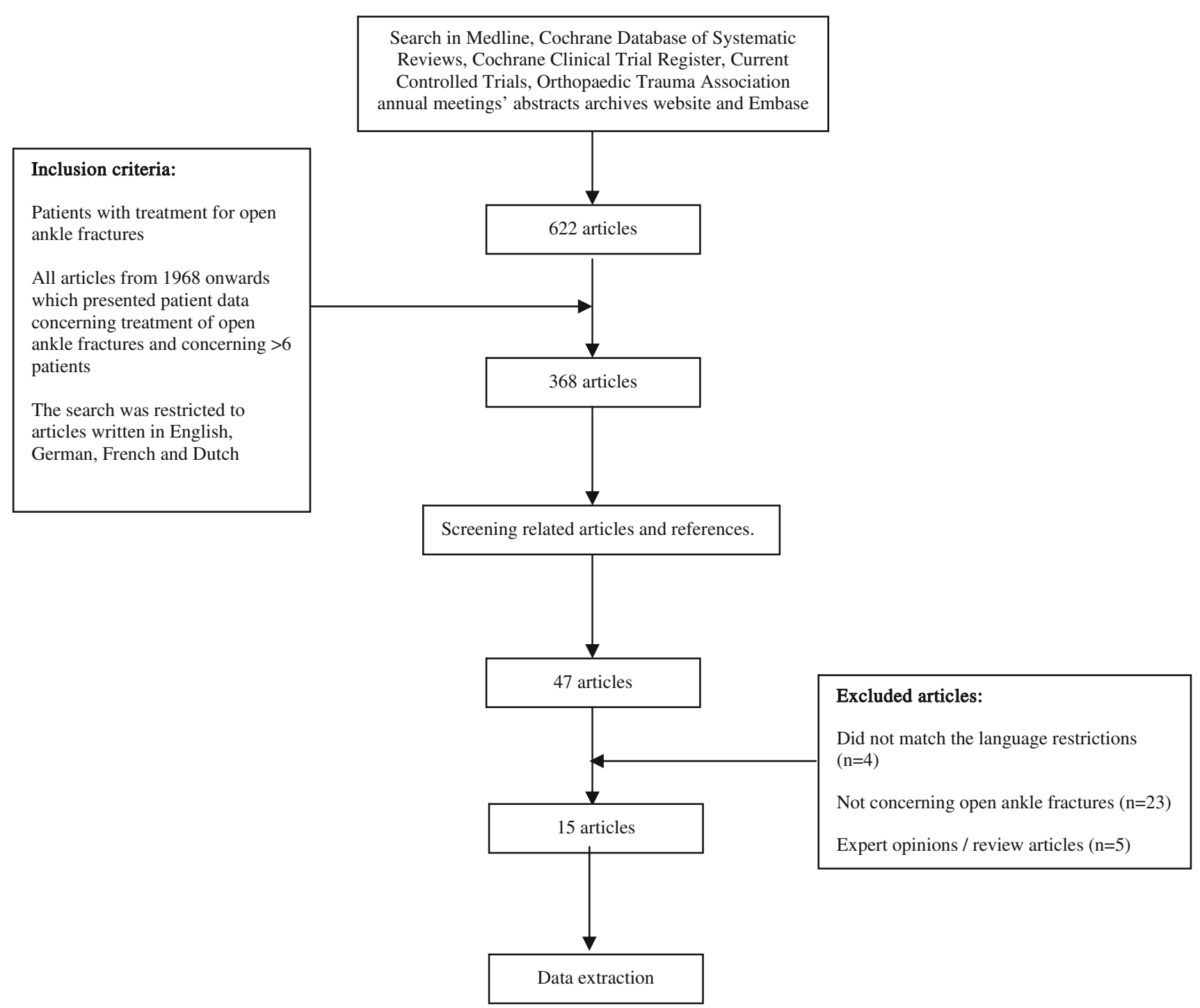

Fig. 1 Flowchart summarizing the selection of relevant articles

Table 2 Pubmed/Medline search strategy

(((( (“open fracture”[tw] OR “open fractures”[tw]) OR (“Fractures, Open”[Mesh]) OR (“complex fractures”[tw] OR “complex fracture” [tw]) OR (“compound fracture"[tw] OR "compound fractures"[tw])) AND ((ankle[tw] OR ankles[tw]) OR (“Ankle”[Mesh]))) OR ((“"Fractures, Bone"[Mesh:noexp]) OR ("bone fracture"[tw] OR "bone fractures"[tw])) AND (open[tw]) AND ((ankle[tw] OR ankles[tw]) OR (“Ankle”[Mesh]))) OR (((“malleolar fracture”[tw] OR “malleolar fractures”[tw]) OR (“ankle fracture”[tw] OR "ankle fractures”[tw])) AND (open[tw]))) AND (("Treatment Failure"[Mesh]) OR (treatment[tw]) OR ("operative procedure"[tw] OR "operative procedures"[tw]) OR ("Surgical Procedures, Operative"[Mesh:noexp]) OR ("debridement”[MeSH Terms] OR "debridement”[tw]) OR ("Irrigation"[ Mesh:noexp]) OR (Irrigation[tw]) OR (lavage[tw]) OR (“Anti-Bacterial Agents”[Mesh:noexp]) OR (antibiotics[tw]) OR ("Fracture Fixation, Internal"[Mesh]) OR ("internal fracture fixation"[tw]) OR (osteosynthesis[tw])))

articles for full review. From the full text, using the abovementioned criteria two reviewers $(\mathrm{CH}, \mathrm{MB})$ independently selected articles for inclusion in this review. Disagreement was resolved by group discussion with arbitration by a third author (CZ) where differences remained.

The data from the included studies were extracted (SK) and verified by a second author (CZ). Disagreement was resolved in a consensus meeting or by third party (SK) adjudication when necessary. Studies were not blinded for author, affiliation and source [8, 9]. If necessary, authors were contacted to acquire further information on methodology and data.

It was the initial intention of the authors to use a strict methodology for paper analysis, focusing on objectively 
Table 3 Level of evidence

Level I: high-quality prospective randomized clinical trial

Level II: prospective comparative study

Level III: retrospective case control study

Level IV: case series

Level V: expert opinion

measurable variables, separate evaluation of different Danis-Weber and Lauge-Hansen classifications and randomized controlled trials. These standards had to be abandoned, however, as almost none of the available papers fulfilled the above-mentioned criteria and data could not be pooled.

The methodological quality of included studies was assessed by assigning Levels of Evidence as previously defined by the Center for Evidence-Based Medicine (CEBM) [8], referred to in Table 3.

\section{Results}

Fifteen studies met the inclusion criteria, including 498 patients. The number of included patients varied from 11 to 64. An overview of the included studies is shown in Table 4. The The publication dates span 35 years. In 1973, Paul et al. published the earliest and in 2008, Lee et al. published the most recent study. There were 2 prospective studies and 13 retrospective studies. All articles were case series and were classified as level IV evidence. There was a wide variation in ankle fracture types from unimalleolar to trimalleolar fractures. The treatments varied from nonoperative to extended debridement, irrigation, intravenous antibiotics, ORIF and delayed closure. There was a wide variation in follow-up ranging from 10 [10] to 90 [11] months. Different inclusion criteria (different treatment protocols, trauma-to-treatment intervals and postoperative rehabilitation protocols) prohibited a statistical evaluation and comparison between the studies. Fifteen articles, concerning 498 patients with treatment of an open ankle fracture, were identified. In 373 cases, open ankle fractures were treated by immediate internal fixation. In 125 cases, a conservative treatment or delayed/other fixation treatment was followed. Of those patients treated by immediate internal fixation, $81 \%$ had a satisfactory result. Poor results (15\%) were most commonly due to non-anatomic reductions, articular surface damage or deep infection. When conservative treatment was followed, $76 \%$ had satisfactory results. Different scoring systems for outcome were used by different authors; hence, we can only refer to outcomes as "good", "satisfactory" or "poor". The most reported complications after immediate internal fixation were deep infection (8\%) and skin necrosis (14\%).

\section{Discussion}

The complexity of open ankle fractures warrants treatment by surgeons who are experienced in this field and have the ability to collaborate with other surgical disciplines to obtain the best possible treatment [12]. In accordance with the British guidelines, we advised that if this experience was not available, prompt referral was advised. Provisional stabilization with an external fixator is the optimal treatment of damage control before referral to another more experienced surgeon [12].

Heterogeneity of data made it impossible to carry out pooling of results. Furthermore, different scoring systems for functional outcome were used, which made quantitative analysis of functional outcomes impossible.

Timing of operative treatment

Emergency operative treatment has long been the standard of care for open fractures. The origin of the so-called "sixhour rule" is unclear; it is probably based on old studies.

Based on the available evidence it is not possible to reject or confirm this rule because there are studies in favor of and against this "golden" rule. Some authors suggest that operative debridement might not be necessary for lowgrade open fractures [13-15]. Poor functional results seem to be associated with inability to achieve anatomical reduction and postoperative loss of reduction of the ankle fracture rather than delayed operative treatment [16, 17]. In our review, the timing of operative treatment ranged from 2 to $19 \mathrm{~h}$. Most of the studies do not report on timing of operative treatment with respect to infection rates, but there is a trend that type of soft tissue damage (high-energy injuries, crush-type soft tissue injury around the ankle, grade III open injuries) is the determinant of postoperative complications as opposed to timing of treatment [16, 17] as long as this is within $24 \mathrm{~h}$ (Grade C) (Table 5).

\section{Wound irrigation}

Irrigation is a key component of the effort to prevent infection after open fracture, as it serves to decrease bacterial load and to remove foreign bodies. Although many guidelines call for "copious" amounts of irrigation, there are little data on exactly how much volume should be used in the lavage of open fracture wounds. With regard to the delivery of irrigation, high-pressure pulsatile lavage is most effective for the removal of bacteria and other contaminants. There is increasing evidence from animal and in vitro studies that 


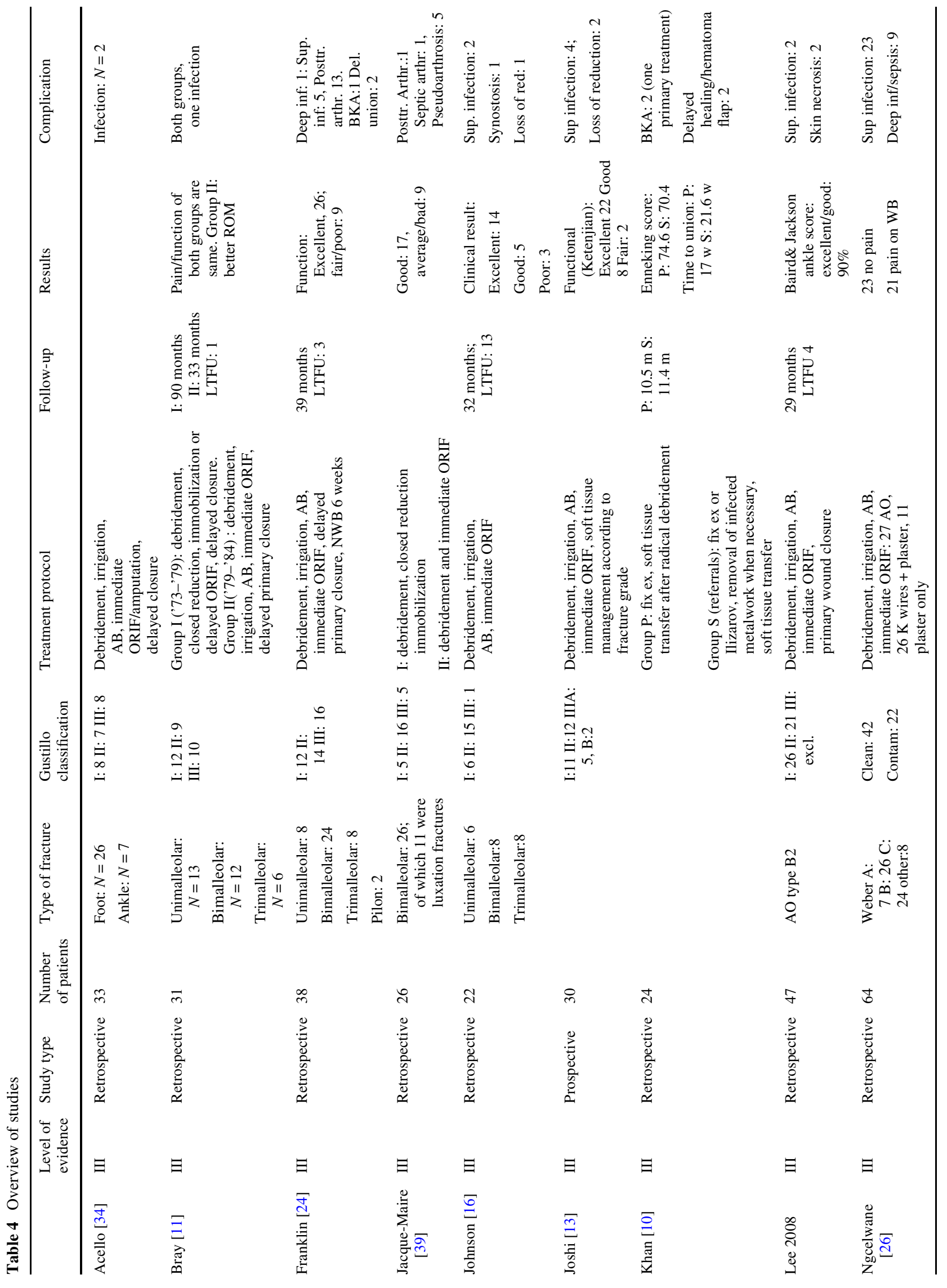




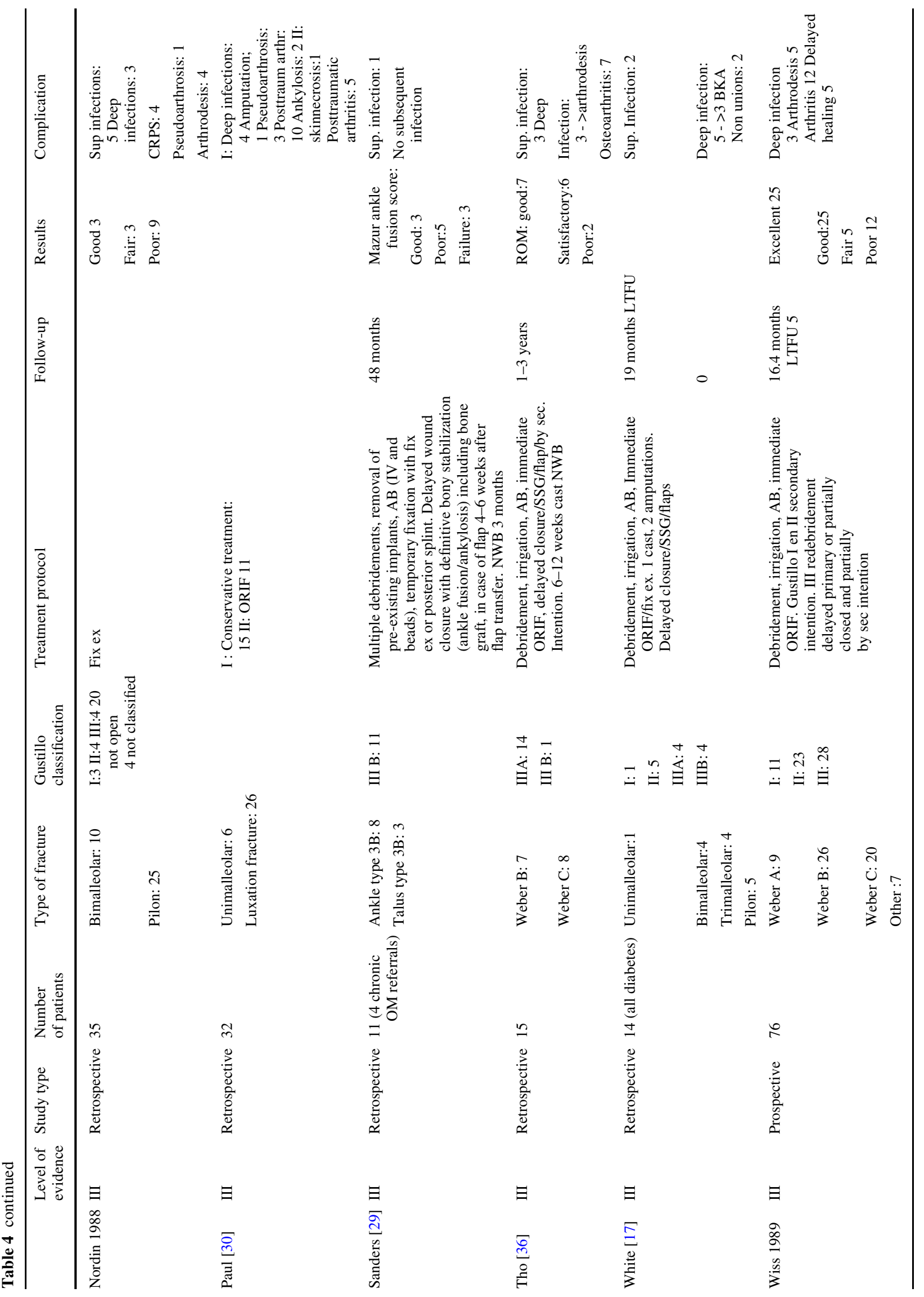


Table 5 Grades of recommendation (given to various treatment options based on the level of evidence supporting that treatment)

Grade A: treatment options are supported by strong evidence (consistent with level I or II studies)

Grade B: treatment options are supported by fair evidence (consistent with level III or IV studies)

Grade C: treatment options are supported by either conflicting or poor-quality evidence (level IV studies)

Grade D: when insufficient evidence exists to make a recommendation

high-pressure pulsatile lavage may have deleterious side effects [11, 18-21]. These effects include more macroscopic bone damage, reduced mechanical strength at short-term follow-up and increased depth of bacterial penetration into muscle [11, 18-21]. However, there is no clear clinical evidence in favor of high- or low-pressure pulsatile lavage. Okike and Bhattacharyya concluded that it was not possible to recommend any particular additive for the irrigation of open fracture wounds [1].

\section{Role of fixation}

Fixation of open fractures has a number of beneficial effects, including protection of soft tissues from additional injury by fracture fragments, improvement of wound care and tissue healing, promotion of early mobilization and rehabilitation, and possibly even reduction of the risk of infection [22]. The mode of fixation of open fractures has historically been a topic of debate. In our review, immediate open reduction and rigid internal fixation of open ankle fractures is safe and leads to good functional outcome (Grade C). It leads to shorter hospital stay and less joint stiffness when compared with conservative or delayed fixation [11]. Isolated medial malleolar fractures can be fixed by a single screw or without screw fixation [12, 23]. Even in grade III open ankle fractures, rigid internal fixation is associated with good outcome. Fibular plating is not necessary at index surgery when the ankle is stabilized by external fixation [23]. Deep infection leads to poor outcome and, in one study, all cases with deep infection required ankle arthrodesis [17]. Deep infection should be avoided by preserving the soft tissues surrounding the fracture site by lavage, debridements (repeated on demand) and early administration of antibiotics [13, 19]. Only when there is inadequate soft tissue to cover osteosynthesis materials, external fixation should be considered [17]. Poor outcome is not only caused by deep infection. A large amount of bone loss and articular cartilage damage caused by the injury, inability to achieve articular reduction at operation and postoperative loss of anatomical reduction are associated with painful secondary osteoarthritis $[16,17,24]$. It is imperative to achieve a congruous ankle mortise to prevent early degenerative ankle joint changes [17, 25], and failure to achieve rigid fixation may even lead to higher deep infection rates [26].

\section{Wound coverage and closure}

An anterior soft tissue defect resulting from direct injury of hyper-plantar flexion of the ankle can be associated with injury of anterior tibial vessels and disruption of the extensor tendons [12]. Reconstruction of the tendons by interposition grafts or tenodesis, and the extensor retinaculum is advised [12]. Previously, the closure of wounds of open fractures were delayed to prevent infection with contaminating organisms. This strategy remains the generally accepted approach in settings characterized by substantial contamination. Today, many orthopedic surgeons consider earlier closure of open fracture wounds that have been adequately debrided. The trend toward early closure of open fractures conflicts with recommendations for routine debridement of open fractures [27]. There are a number of methods for achieving closure, including direct suturing, split skin grafting and the use of free or local muscle flaps. The optimal method depends on a number of factors, including the location of the defect, its size, associated injuries and patient characteristics such as the amount of function retained and the desired level of function [1].

There is no consensus on the treatment of Grade I and II open wounds. Whereas most authors advocate delayed primary closure for most Grade I and II open wounds, some report leaving these to heal by secondary intention [13, 25] or to close Grade I wounds primarily as long as tension of the wound edges is avoided [6]. Some studies do not report on closure technique after managing an open ankle fracture at all [11, 26, 28, 29]. Most authors have chosen to mention Gustilo grade rather than closure technique when discussing cases of infection, which makes it difficult to correlate infection with closure technique. Consensus is that all gross contamination should be cleaned in the emergency department, after which grade I and II open wounds can be left to heal by secondary intention or delayed primary closure (Grade C).

Managing grade III open ankle fractures remains a challenging task. Infection is a major threat to successful treatment. Soft tissue coverage of the ankle is limited due to the lack of muscle around it. Free flaps are the only option for covering large defects around the ankle [17]. Primary closure of grade III open ankle fractures is discouraged (grade C). Early institution of surgical debridement and antibiotics seems key to successful management of grade III ankle fractures, followed by delayed closure after 2-5 days when signs of infection are absent. When this is not possible, split 
skin grafts can be used to cover skin defects on well-vascularised beds and free flaps to cover larger defects [17, 24] (grade C).

\section{Use of antibiotics}

Antibiotic use has been considered the standard of care since 1974, when Patzakis et al. reported their results of the effect of a first-generation cephalosporin for the management of open fractures [30]. The benefit of antibiotics was confirmed by a recent Cochrane systematic review [31], which showed that the administration of antibiotics after an open fracture reduced the risk of infection by $59 \%$ (relative risk, 0.41; 95\% confidence interval, 0.27-0.63). Okike and Bhattacharyya do not recommend the routine use of cultures either before or after debridement because the organisms that are found to be contaminating an open fracture on presentation do not represent the microbes that will eventually cause infection [32]. While there is ample data supporting the administration of antibiotics after open fracture, evidence indicating an optimal regimen is lacking [32]. There is agreement that a first-generation cephalosporin should be administered in patients with open fractures $[6,15]$. Local antibiotic therapy is a useful adjunct to systemic antibiotics in the management of open fractures [32]. Whether this applies to the treatment of open ankle fractures could not be evinced from the articles in this review.

Timing of antibiotic administration plays an important role. The rate of infection increases when antibiotics are commenced more than $3 \mathrm{~h}$ post-injury [33].

The duration of antibiotic treatment differed greatly in the studies of our review, ranging from 24 to $48 \mathrm{~h}[9,16$, $24,28], 3$ days [26, 34, 35] to 5-7 days [13, 36]. One author advocates administering antibiotics until discharge from hospital [29]. It is not possible to extract data which allow a correlation between postoperative infection and duration of antibiotic treatment.

\section{Additional therapies}

Non-unions of ankle fractures are rare [37], so adjunctive therapy such as bone grafting as used in tibia and femur fractures is not beneficial. The use of tourniquets during operation is discouraged [34], but no firm evidence is available to support this.

\section{Conclusion}

There is a lack of high-quality literature concerning the (operative) treatment of patients with open ankle fractures. Most authors reported good results after performance of their treatment protocol. On basis of the existing literature, we come to the following guidelines.

- All gross debris and contamination should be removed in the emergency department (Grade C).

- Cephalosporins should be administered in the emergency department without delay. It is not imperative that wound swabs be taken before administering antibiotics as initial swabs taken do not represent the microbes that eventually cause infection.

- There is no evidence regarding the optimal duration of antibiotic treatment.

- Patients should be taken to the theater within $24 \mathrm{~h}$ (Grade C).

- After thorough debridement of all devitalized tissues, irrigation of the wound should be carried out with caution as this may also have deleterious effects on bone and healthy soft tissue (Grade C).

- There is no firm evidence against the use of tourniquets (Grade C).

- Rigid internal fixation should be carried out with the aim of restoring anatomy of the ankle mortise and preventing long-term secondary degenerative changes resulting in pain and stiffness. Only when there is inadequate soft tissue to cover osteosynthesis materials, external fixation should be considered (Grade C).

- Grade I wounds may be closed, primarily if the wound is not under tension, or left open to heal by secondary intention (Grade C).

- Grade II wounds should be left to heal by secondary intention, or be closed primarily at a later time after postoperative infection has been ruled out (Grade C).

- Grade III open ankle injuries should be left open and managed postoperatively by the use of skin grafts or flee flaps (Grade C).

Future studies should focus on the choice and duration of antibiotic treatment, the amount and intensity of irrigation of soft tissues around the ankle and the type of irrigation solution to be used.

Acknowledgments The authors are grateful to Albertine van Horssen for her help with the systematic search and collecting the required articles. The authors did not receive any outside funding or grants in support of their research or for preparation of this work. Neither they nor a member of their immediate families received payments or other benefits, or a commitment or agreement to provide such benefits from a commercial entity. Non-commercial entity paid or directed, or agreed to pay or direct, any benefits to any research fund, foundation, division, center, clinical practice or other charitable or nonprofit organizations, with which the authors, or a member of their immediate families are affiliated or associated.

Open Access This article is distributed under the terms of the Creative Commons Attribution Noncommercial License which permits any noncommercial use, distribution, and reproduction in any medium, provided the original author(s) and source are credited. 


\section{References}

1. Olerud S, Karlstrom G, Danckwardt-Lilliestrom G (1978) Treatment of open fractures of the tibia and ankle. Clin Orthop Relat Res (136):212-224

2. Gustilo RB, Anderson JT (1976) Prevention of infection in the treatment of one thousand and twenty-five open fractures of long bones: retrospective and prospective analyses. J Bone Joint Surg Am 58:453-458

3. Gustilo RB, Mendoza RM, Williams DN (1984) Problems in the management of type III (severe) open fractures: a new classification of type III open fractures. J Trauma 24:742-746

4. Brumback RJ, Jones AL (1994) Interobserver agreement in the classification of open fractures of the tibia. The results of a survey of two hundred and forty-five orthopaedic surgeons. J Bone Joint Surg Am 76:1162-1166

5. Horn BD, Rettig ME (1993) Interobserver reliability in the Gustilo and Anderson classification of open fractures. J Orthop Trauma 7:357-360

6. Olson SA, Finkemeier CG, Moehring ND (2001) Open fractures. In: Bucholz RW, Heckman JD (eds) Rockwood and Greene's fractures in adults, 5th edn. Lippincott, Williams and Wilkins, Philadelphia, pp 285-318

7. Moher D, Cook DJ, Eastwood S, Olkin I, Rennie D, Stroup DF (1999) Improving the quality of reports of meta-analyses of randomised controlled trials: the QUOROM statement. Quality of reporting of meta-analyses. Lancet 27, 354(9193):1896-1900

8. Jadad AR, Moore A, Carroll D et al (1996) Assessing the quality of reports of randomised controlled trials: is blinding necessary? Contr Clin Trials 17:1-12

9. Stiehl JB (1990) Open fractures of the ankle joint. Instr Course Lect 39:113-117

10. Khan U, Smitham P, Pearse M, Nanchahal J (2007) Management of severe open ankle injuries. Plast Reconstr Surg 119(2):578-589

11. Bray TJ, Endicott M, Capra SE (1989) Treatment of open ankle fractures. Immediate internal fixation versus closed immobilization and delayed fixation. Clin Orthop Relat Res (240):47-52

12. Herscovici D Jr, Scaduto JM, Infante A (2007) Conservative treatment of isolated fractures of the medial malleolus. J Bone Joint Surg Br 89(1):89-93

13. Joshi D, Singh D, Ansari J, Lal Y (2006) Immediate open reduction and internal fixation in open ankle fractures. J Am Podiatr Med Assoc 96(2):120-124

14. Patrick JH, Smelt GJ (1977) Surgical progress-100 years ago. An assessment of Listerism at St Thomas's Hospital, London. Ann R Coll Surg Engl 59:456-462

15. Zalavras CG, Patzakis MJ, Holtom PD, Sherman R (2005) Management of open fractures. Infect Dis Clin North Am 19:915-929

16. Johnson EE, Davlin LB (1993) Open ankle fractures. The indications for immediate open reduction and internal fixation. Clin Orthop Relat Res (292):118-127

17. White CB, Turner NS, Lee GC, Haidukewych GJ (2003) Open ankle fractures in patients with diabetes mellitus. Clin Orthop Relat Res (414):37-44

18. Bhandari M, Schemitsch EH, Adili A, Lachowski RJ, Shaughnessy SG (1999) High and low pressure pulsatile lavage of contaminated tibial fractures: an in vitro study of bacterial adherence and bone damage. J Orthop Trauma 13:526-533

19. Boyd JI III, Wongworawat MD (2004) High-pressure pulsatile lavage causes soft tissue damage. Clin Orthop Relat Res 427:13-17

20. Dirschl DR, Duff GP, Dahners LE, Edin M, Rahn BA, Miclau T (1998) High pressure pulsatile lavage irrigation of intraarticular fractures: effects on fracture healing. J Orthop Trauma 12:460-463
21. Hassinger SM, Harding G, Wongworawat MD (2005) Highpressure pulsatile lavage propagates bacteria into soft tissue. Clin Orthop Relat Res 439:27-31

22. Yang EC, Eisler J (2003) Treatment of isolated type I open fractures: is emergent operative debridement necessary? Clin Orthop Relat Res 410:289-294

23. British orthopaedic Association and British Association of Plastic, Reconstructive and Aesthetic Surgeons Standard for Trauma (2009) The management of severe open lower limb fractures. Chapter 15: Open fractures of foot and ankle

24. Franklin JL, Johnson KD, Hansen ST Jr (1984) Immediate internal fixation of open ankle fractures. Report of thirty-eight cases treated with a standard protocol. J Bone Joint Surg Am 66(9):1349-1356

25. Worlock P, Slack R, Harvey L, Mawhinney R (1994) The prevention of infection in open fractures: an experimental study of the effect of fracture stability. Injury 25:31-38

26. Ngcelwane MV (1990) Management of open fractures of the ankle joint. Injury 21(2):93-96

27. Orcutt S, Kilgus D, Ziner D (1988) The treatment of low-grade open fractures without operative debridement. Read at the Annual Meeting of the Orthopaedic Trauma Association, Dallas, TX, USA

28. Lee YS, Chen SW (2009) Lateral fixation of open AO type-B2 ankle fractures: the Knowles pin versus plate. Int Orthop 33(4):1135-1139

29. Sanders R, Pappas J, Mast J, Helfet D (1992) The salvage of open grade IIIB ankle and talus fractures. J Orthop Trauma 6(2):201-208

30. Paul D (1973) Conservative and surgical therapy of open malleolar fractures. Zentralbl Chir 2, 98(44):1589-1593

31. Gosselin RA, Roberts I, Gillespie WJ (2004) Antibiotics for preventing infection in open limb fractures. Cochrane Database Syst Rev 1:CD003764

32. Okike K, Bhattacharyya $T$ (2006) Trends in the management of open fractures. A critical analysis. J Bone Joint Surg Am 88(12):2739-2748

33. Patzakis MJ, Harvey JP Jr, Ivler D (1974) The role of antibiotics in the management of open fractures. J Bone Joint Surg Am 56:532-541

34. Acello AN, Wallace GF, Pachuda NM (1995) Treatment of open fractures of the foot and ankle: a preliminary report. J Foot Ankle Surg 34(4):329-346

35. Wiss DA, Gilbert P, Merritt PO, Sarmiento A (1988) Immediate internal fixation of open ankle fractures. J Orthop Trauma 2(4):265-271

36. Tho KS, Chiu PL, Krishnamoorthy S (1994) Grade III open ankle fractures-a review of the outcome of treatment. Singap Med J 35(1):57-58

37. McGonagle L, Ralte P, Kershaw S (2010) Non-union of Weber B distal fibula fractures: a case series. Foot Ankle Surg 16(3):63-67

38. Adili A, Bhandari M, Schemitsch EH (2002) The biomechanical effect of high pressure irrigation on diaphyseal fracture healing in vivo. J Orthop Trauma 16:413-417

39. Jacquemaire B, Babin S, Katzner M, Calmes E, Schvingt E (1976) Treatment of open malleolar fractures. Apropos of a series of 26 cases. J Chir (Paris) 112(6):419-430

40. Lister J (1867) On a new method of treating compound fracture, abscess, etc. Lancet 1:326, 357, 387, 507

41. Patzakis MJ, Harvey JP Jr, Ivler D (1974) The role of antibiotics in the management of open fractures. J Bone Joint Surg Am 56:532-541

42. Schulz KF, Chalmers I, Grimes DA et al (1994) Assessing the quality of randomisation from reports of controlled trials published in obstetrics and gynaecology journals. JAMA 272:125-128 